\title{
Staurosporine and cytochalasin $D$ induce chondrogenesis by regulation of actin dynamics in different way
}

\author{
Minjung Kim ${ }^{1}$, Kyung Song ${ }^{2}$, \\ Eun-Jung $\mathrm{Jin}^{3}$ and Jongkyung Sonn ${ }^{1,4}$ \\ ${ }^{1}$ Department of Biology \\ College of Natural Sciences \\ Kyungpook National University \\ Daegu 702-701, Korea \\ ${ }^{2}$ Department of Pharmacy \\ College of Pharmacy \\ ${ }^{3}$ Department of Biological Science \\ College of Natural Sciences \\ Wonkwang University \\ Iksan 570-749, Korea \\ ${ }^{4}$ Corresponding author: Tel, 82-53-950-7368; \\ Fax, 82-53-953-3066; E-mail, sonnjk@knu.ac.kr \\ http://dx.doi.org/10.3858/emm.2012.44.9.059
}

Accepted 22 May 2012

Available Online 8 June 2012

Abbreviations: CD, cytochalasin D; CIN, chronophin; F-actin, filamentous actin; G-actin, globular actin; MLC, myosin light chain; ROCK, Rho-associated kinase; SSH, slingshot; STSN, staurosporine; TESK, testicular kinases

\begin{abstract}
Actin cytoskeleton has been known to control and/or be associated with chondrogenesis. Staurosporine and cytochalasin $\mathrm{D}$ modulate actin cytoskeleton and affect chondrogenesis. However, the underlying mechanisms for actin dynamics regulation by these agents are not known well. In the present study, we investigate the effect of staurosporine and cytochalasin $D$ on the actin dynamics as well as possible regulatory mechanisms of actin cytoskeleton modulation. Staurosporine and cytochalasin $D$ have different effects on actin stress fibers in that staurosporine dissolved actin stress fibers while cytochalasin $D$ disrupted them in both stress forming cells and stress fiber-formed cells. Increase in the G-/F-actin ratio either by dissolution or disruption of actin stress fiber is critical for the chondrogenic differentiation. Cytochalasin $D$ reduced the phosphorylation of cofilin, whereas staurosporine
\end{abstract}

showed little effect on cofilin phosphorylation. Either staurosporine or cytochalasin $\mathrm{D}$ had little effect on the phosphorylation of myosin light chain. These results suggest that staurosporine and cytochalasin D employ different mechanisms for the regulation of actin dynamics and provide evidence that removal of actin stress fibers is crucial for the chondrogenic differentiation.

Keywords: actin cytoskeleton; chondrogenesis; cytochalasin D; actins; myosin light chains; staurosporine

\section{Introduction}

Dynamic actin cytoskeleton is essential for diverse cellular processes such as the driving cell shape changes, cellular motility, adhesion, cytokinesis, and endocytosis (Pollard and Cooper, 2009). The significance of cell shape in the chondrogenesis has been recognized by the findings of cell shape transition from stellate, fibroblastic-like to rounded during condensation stage of limb bud mesenchyme (Wezeman, 1998) and of association of changes in morphology with a gradual loss of collagen type II and proteoglycan synthesis (von der Mark and von der Mark, 1977). Rounded cells are also observed in the cartilage-containing cell aggregates of the micromass culture which mimics the condensation and differentiation events that normally occur during embryonic cartilage formation in vivo (Ahrens et al., 1997).

Cellular condensation during which cell shape change occurs is a complicated process that are regulated by multiple cellular signaling molecules (DeLise et al., 2000) and therefore, it could be hard to explore the mechanisms of cell shape changes and the role of cell shape and/or cytoskeleton in the chondrogenesis using micromass culture system. Single cell culture systems would provide good models for the study of role of cell shape and/or cytoskeleton in chondrogenesis.

Chondrogenesis is induced in single cell culture systems such as suspension culture (Solursh et al., 1982) and three-dimensional culture systems using polymers, hydrogels or micromass culture 
(Tortelli and Cancedda, 2009). The latter culture systems mimic tissues, resemble their organization, their mechanical properties and their physiological response to different stimuli.

A method that belongs to other side of category in inducing chondrogenesis of single cells is to use actin filaments-modulating chemical agents. Cytochalasin D, a blocker of actin polymerization and elongation of actin, has been extensively used for the study of chondrogenesis. It has been known to induce chondrogenesis of limb mesenchymal cultures (Zanetti and Solursh, 1984; Lim et al., 2000) and stimulate chondrogenesis of dedifferentiated chondrocytes (Brown and Benya, 1988; Loty et al., 1995). Staurosporine, a broad spectrum protein kinase inhibitor, disrupts the actin stress fibers and restores the differentiated functions of dedifferentiated chondrocytes (Borge et al., 1997). However, the underlying mechanisms by which these agents modulate actin cytoskeleton are poorly understood.

Actin binding proteins regulate disassembly and assembly of actin filaments by sequestering $\mathrm{G}$-actin and by depolymerizing actin. Formation of actin stress fibers is induced by myosin light chain (MLC) phosphorylation which regulates the activity of non-muscle myosin type II (Totsukawa et al., 2000; Vicente-Manzanares, 2009). Cofilin binds to both monomeric and filamentous actin and increase actin dynamics by depolymerizing filaments form their pointed ends (Paavilainen et al., 2004). The activity of cofilin is regulated by phosphorylation of Serine 3. Phosphorylation of cofilin abolishes the ability of cofilin to bind to F-actin leading to loss of its ability to depolymerize F-actin (Agnew et al., 1995; Moriyama et al., 1996; Bamburg and Bernstein, 2010).

At the top of signaling pathway that regulate actin dynamics is Rho family of GTPase (Etienne-Manneville and Hall, 2002). RhoA has been shown to be involved in chondrogenesis (Kumar and Lassar, 2009; Kim et al., 2012).

In the present study, we employed two different chemicals, staurosporine and cytochalasin D to modulate actin filaments. First, we tested whether staurosporine induces differentiation of undifferentiated mesenchymal cells into chondrocytes. Second, we compared the effect of staurosporine and cytochalasin $D$ on the actin dynamics and actin regulatory proteins including MLC and cofiln.

\section{Results}

\section{Staurosporine and cytochalasin D induce chondrogenesis of single mesenchymal cell and reorganization of actin filaments}

The changes in the F-actin cytoskeleton of monolayer culture of chick embryo wing bud mesenchymal cells were demonstrated by phalloidin staining. The stress fibers began to appear on day one of culture and became abundant and thicker on day two (Figure 1A). These cells remained undifferentiated state based on the immunofluorescence and western blot assay for collage type II. In the staurosporine-treated cells, stress fibers disappe-

A

\begin{tabular}{|c|c|c|c|c|c|c|c|c|}
\hline \multicolumn{3}{|c|}{ Con } & \multicolumn{3}{|c|}{ STSN } & \multicolumn{3}{|c|}{$C D$} \\
\hline Type II & Phalloidin & Merge & Type II & Phalloidin & Merge & Type II & Phalloidin & Merge \\
\hline $\mathbf{a}$ & b & C & g & h & i & $\mathrm{m}$ & & \\
\hline d & & & & & & & & \\
\hline
\end{tabular}

B

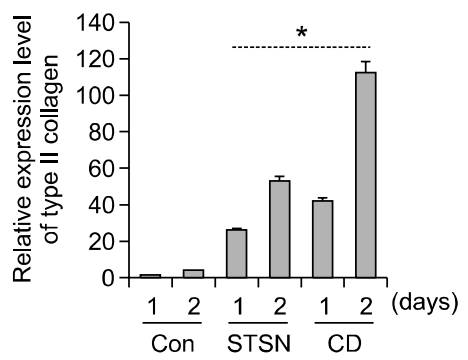

Figure 1. Effect of staurosporine and cytochalasin $D$ on the actin cytoskeleton and chondrogenesis. (A) Cell were cultured in the absence (a-f) or presence of staurosporine (STSN, $5 \times 10^{-9} \mathrm{M}$ ) or cytochalasin $\mathrm{D}(\mathrm{CD}, 1 \mu \mathrm{g} / \mathrm{ml})$ for 1 (upper panel) or 2 (lower panel) days at low density and stained for F-actin (Phalloidin) and type II collagen (Type II). (B) The transcription level of type II collagen was analyzed by real-time PCR at 1 and 2 day of culture (right panel) and the translational level of collagen type II and Sox9 was analyzed by Immunoblotting at 2 day of culture (left panel). ${ }^{*} P<0.05$ compared to control. The data shown are representative of at least four independent experiments. 
ared and F-actin assumed a dispersed distribution from the day one of culture. Staurosporine treatment for 2 days induced the expression of type II collagen. Cytochalasin D treatment also resulted in disintegration of the stress fibers but F-actin exhibited an aggregated pattern. Cytochalasin D-treated cells also expressed collagen type II as in the case of staurosporine. These results are consistent with previous reports (Zanetti and Solursh, 1984; Borge et al., 1997; Lim et al., 2000).

When cells from limb bud mesenchyme were plated on the culture dish, they initially exhibited round form and became flattened as cultures continued. To examine whether staurosporine or cytochalasin D has same actin-dissolving or -disrupting effect on the stress fibers, mesenchymal cells were incubated for two days and staurosporine or cytochalasin $D$ was treated for another 2 days. As shown in Figure 2, cells cultured for 4 days has well-developed stress fibers. Staurosporine dissol-

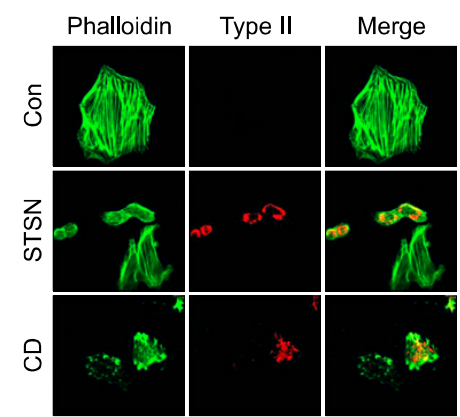

Figure 2. Staurosporine and cytochalasin $D$ disrupt existing actin stress fibers and induce chondrogenesis. Cell were cultured in F12 media supplemented with $10 \%$ FBS for 2 days and cultured for another two days in the absence or presence of staurosporine (STSN, $5 \times 10^{-9} \mathrm{M}$ ) or cytochalasin $\mathrm{D}(\mathrm{CD}, 1 \mu \mathrm{g} / \mathrm{ml})$ and stained for F-actin (Phalloidin) and collagen type II (Type II). ved actin stress fibers and induced chondrogenesis. Cells which have still stress fibers, even thin and small in number, were not stained for collagen type II. Cytochalasin D also disrupted actin stress fibers and induced chondrogenesis. Some cells of which stress fibers were disrupted by cytochalasin $D$ did not differentiate into chondrocytes. These results indicate that staurosporine and cytochalasin $D$ are able to block formation of new stress fibers and dissolve or disrupt existing stress fibers leading to chondrogenic differentiation.

\section{Relative amount of F- and G-actin and F/G-actin ratio}

To quantify effects of staurosporine and cytochalasin D on actin dynamics of mesenchymal cell, we measured the relative amount of filamentous $(F)$-actin, globular (G)-actin, and relative abundance of F-actin compared with G-actin (F/G-actin ratio). As shown in Figure 3, the relative amount of F-actin fraction was not significantly changed by the two agents. These results were also confirmed by the analysis of F-actin amount with anti-F-actin antibody (Figure 3) consistent with the analysis of F-actin immunofluorescence with phalloidin as shown in Figure 1. F-actin which was dissolved by staurosporine or cytochalasin D, showed a diffused or spotted distribution, respectively. The relative amount of G-actin was decreased with staurosporine treatment and increased with cytochalasin $D$ treatment, which were reflected in the $F / G$ actin ratio. Both of staurosporine and cytochalasin $d$ reduced $\mathrm{F}$-actin/G-actin ratio with more effect by cytochalasin D (Figure 3B).

\section{Staurosporine and cytochalasin $\mathrm{D}$ have different effect on the phosphorylation of cofilin}

To find the factors which regulate actin dynamics
A

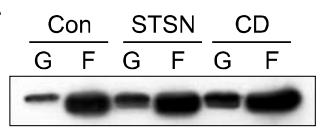

B

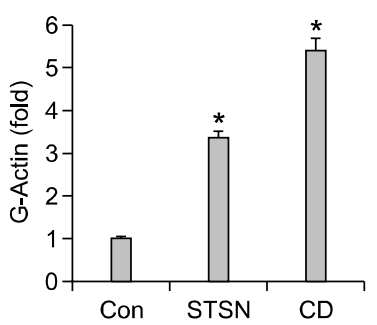

C

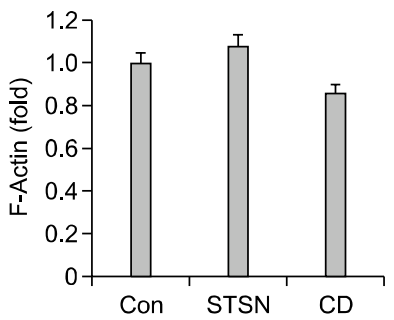

D

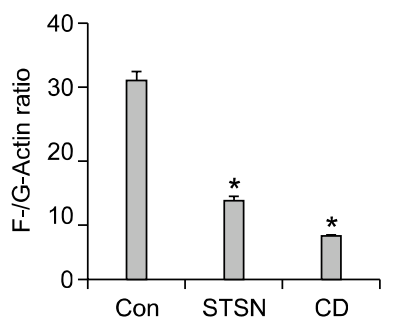

Figure 3. Effect of staurosporine and cytochalasin $\mathrm{D}$ on the actin dynamics. Cells were cultured in the absence or presence of staurosporine (STSN) or cytochalasin D (CD) for 2 days at low density. F- and G-actin fractions were subjected to Western blotting $(A)$. Densitometric evaluation of the G-actin (B), F-actin (C), and F-/G-actin ratio (D). ${ }^{*} P<$ 0.05 compared to control. The data shown are representative of at least four independent experiments. 
A
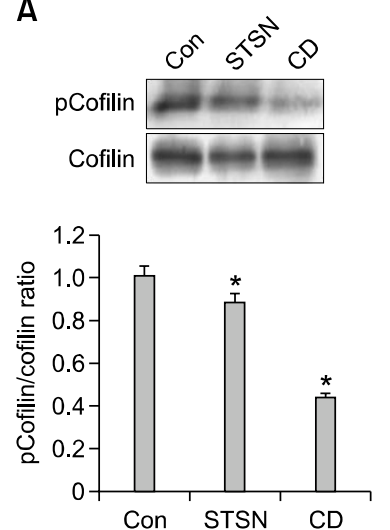

B
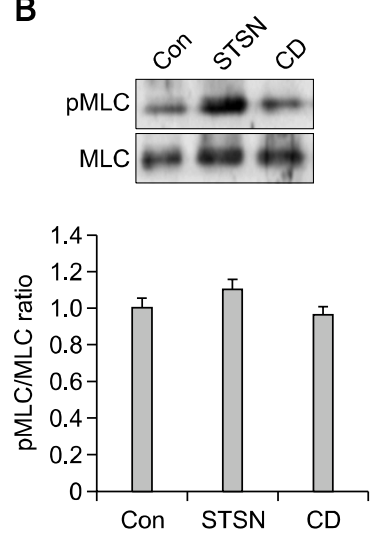

Figure 4. Effect of staurosporine and cytochalasin $D$ on the phosphorylation of cofilin and MLC. Cell were cultured in the presence of staurosporine $\left(5 \times 10^{-9} \mathrm{M}\right)$ or cytochalasin $D(1 \mu \mathrm{g} / \mathrm{ml})$ for 2 days in low density. Cell lysates were loaded on SDS-PAGE gel, immunoblotted with cofilin (A) and MLC (B) antibodies and quantified using imaging system. ${ }^{*} P<0.05$ compared to control. The data shown are representative of at least three independent experiments.

during chondrogenesis induction, we examined whether the two drugs influence the phosphorylation of cofilin. As seen in Figure 4, phosphorylation of cofilin was slightly but not significantly decreased in staurosporine-treated cells. On the other hand, cytochalasin D significantly decreased phosphorylation of cofilin. To test other candidates for modulating actin dynamics, phosphorylation of MLC was examined. Neither staurosporine nor cytochalasin D affected phosphorylation of MLC.

\section{Discussion}

In this present study, we employed two different drugs which modulate actin filaments and compared their effects on chondrogenesis, F-/G-actin ratio, and phosphorylation of cofilin and MLC.

The role of staurosporine in the recovery from the dedifferentiated state and prevention of dedifferentiation-like process as well as cytoskeletal changes has been studied in several cells. Staurosporine disrupts actin filaments and redifferentiates chondrocytes defifferentiated by retinoic acid or serial passages (Benya and Padilla, 1986; Borge et al., 1997; Lee et al., 2003) and stimulates expression of collagen type II in fibrochondrocytes (Hoben and Athanasiou, 2008). In this study, we demonstrate that staurosporine induces the differentiation of single mesenchymal cells extending its influence to undifferentiated cells. Cytochalasin D also showed similar chondrogenesis-inducing effect as previously reported.
However, the effects of these drugs on the actin cytoskeleton were somewhat different. While staurosporine dissolves actin filament with slight decrease in the relative amount of $\mathrm{G}$-actin, F-actin, and F-/G-actin ratio compared to the control, cytochalasin $\mathrm{D}$ considerably reduced $\mathrm{G}$-actin content and F-/G-actin ratio.

Actin dynamics is regulated by actin binding proteins such as MLC and cofilin. Cytochalasins interact with the actin filament network by capping the barbed ends, and thereby altering the dynamic properties of microfilaments (Cooper, 1987). It has been proposed that the effects of cytochalasin $D$ on cells result from both a direct interaction of the drug with the actin filament components of cytoskeletal networks and a secondary cellular response (Schliwa, 1982). The latter engages network fragments in an energy-dependent event that leads to the formation of filament foci (Bershadsky et al., 1980). In the present study, we show that cytochalasin $D$ decreases the phosphorylation of cofilin. Cytochalasin B itself has a weak severing effect (Bonder and Mooseker, 1986). However, it has been reported that cytochalasin $D$ reduces phosphorylation of ADF/cofilin which would activate cofilin and thereby depolymerize F-actin (Rueckschloss and Isenberg, 2001). Thus, our results suggest that cytochalasin D promotes F-actin depolymerization by reduction of cofilin phosphorylation in concert with the prevention of F-actin polymerization by its immediate effect on the organization of cytoskeletal networks. Further studies are needed to elucidate the mechanism of the cytochalasin D-induced dephosphorylation of cofilin.

In contrast to the effects of cytochalasin $\mathrm{D}$ on the phosphorylation of cofilin and MLC, staurosporine, in the present study, did not affect phosphorylation of either cofilin or MLC. Staurosporine selectively inhibits protein kinase $C$ at nanomolar concentrations, but it also inhibits a broad range of protein kinases (Tamaoki et al., 1986; Matsumoto and Sasaki, 1989; Yanagihara et al., 1991). Inhibition of some of them including protein kinase $\mathrm{C}$, protein kinase $\mathrm{A}$, and $\mathrm{Ca}^{2+} /$ calmodulin-dependent protein kinase with specific inhibitors did not induce the chondrogenesis of mesenchymal cells (data not shown).

Assembly and morphogenesis of matrix adhesion are regulated by signals from small G-protein Rho family. Activation of Rho is required for the formation of focal contacts and the associated stress fibers (Nobes and Hall, 1995; Rottner et al., 1999). Rho functions by triggering many target molecules that, in turn, initiate cascades of downstream events. Two of the immediate Rho targets, 
Rho-associated kinase (ROCK) and the formin homology protein mDia1 (a mammalian homologue of Drosophila Diaphanous protein) were shown to mediate the effects of Rho on matrix adhesion and the actin cytoskeleton (Watanabe et al., 1999). ROCK controls actin dynamics by regulating MLC phosphorylation directly or indirectly through MLC phosphatase (Amano et al., 2010) or by regulating cofilin phosphorylation through LIM kinases (Maekawa, et al., 1999).

Increasing evidence suggests that RhoA/ROCK pathway plays a negative role in chondrogenesis (Woods et al., 2005; Kumar and Lassar, 2009; Kim et al., 2012). In the present study, staurosporine reduced the activity of RhoA. Recently, we have shown that RhoA regulates ROCK activity but inhibition of ROCK activity neither affects MLC phosphorylation nor induces chondrogenesis and suggested that RhoA uses other pathway than ROCK for the chondrogenic differentiation (Kim et al., 2012). In accordance with these results, staurosporine affects neither the phosphorylation of MLC nor the phosphorylation of cofilin supporting the hypothesis that RhoA does not utilize ROCK pathway in the chondrogenic differentiation as suggested by Kim et al. (2012).

In the present study, cytochalasin $D$ reduced phosphorylation of cofilin. If ROCK does not function in the chondrogenic differentiation of mesenchymal cell as mentioned before, then how could cytochalasin D affect cofilin phosphorylation? There are other kinases than ROCK which are responsible for cofilin phosphorylation such as testicular kinases (TESK) (Toshima et al., 2001; Scott and Olson, 2007). In addition, the phosphatases of the Slingshot (SSH) family and the haloacid dehalogenase phosphatase chronophin (CIN) dephosphorylate cofilin (Huang et al., 2006). It remains to be established whether cytochalasin $D$ regulates these kinases and phosphatases.

Comparing the effect of staurosporine and cytochalasin D, they have some features in common. They disrupted actin stress fibers and induced chondrogenesis of single mesenchymal cells. They also showed some differences. Staurosporine has less effect on F-actin/G-actin ratio and phosphorylation of cofilin than cytochalasin D. In other words, irrespective of the mechanisms by which staurosporine and cytochalasin D use, the common consequence of the treatment of two drugs is disappearance of actin stress fibers and subsequent induction of collagen type II expression.

In addition to the role in the regulation of cellular mechanic through cytoskeletal modulation, dynamic connection between nearly all cellular structures provides a large negatively charged surface on which many signaling molecules including protein, lipid kinases, phospholipases, and GTPases in response to the activation of specific transmembrane receptors (Janmey, 1998). Moreover, mechanical tension guides repositioning of translational components to sites of signal reception resulting in rapid post-transcriptional changes in gene expression (Chicurel et al., 1998). Therefore, disappearance of stress fiber means the disruption of cytoskeletal network and tension developed by cytoskeletal filaments leading to alteration of cell signaling and gene expression. Taken together, our results suggest that dissolution of actin stress fibers, not F-actin/G-actin ratio, is crucial for chondrogenesis.

In summary, the present results showed that staurosporine and cytochalasin $D$ use different mechanisms in the reorganization of actin filaments and chondrogenic differentiation. Our data also suggest that disappearance of actin stress fibers plays a common and important role in chondrogenic differentiation.

\section{Methods}

\section{Materials}

Goat anti-collagen type II polyclonal antibodies were purchased from Santa Cruz Biotechnology (Santa Cruz, CA). Rabbit anti-phospho-MLC (Ser19) polyclonal antibody was bought from Stressgen (Ann Arbor, MI). Anti MLC antibody was from Sigma (St. Louis, MO). Anti-phospho-cofilin 1 (Ser-3) rabbit polyclonal antibody was from ECM Biosciences LLC (Versailles, KY). Anti-cofilin rabbit polyclonal antibody was from Sigma (St. Louis, MO). Alexa fluor 555 anti-goat antibody and Alexa fluor 488 phalloidin were from Invitrogen (Carlsbad, CA). Cytochalasin D was from Enzo Life Sciences (Farmingdale, NY) and staurosporine from Biomol (Plymouth Meeting, PA).

\section{Cell culture}

Mesenchymal cells isolated from stage $23 / 24$ chick embryo wing buds were cultured as previously described (Lim et al., 2000). The isolated limb mesenchyme was incubated with $0.1 \%$ trypsin-collagenase in $\mathrm{Ca}^{2+}-\mathrm{Mg}^{2+}$-free saline-G for $10 \mathrm{~min}$ at $37^{\circ} \mathrm{C}$. After addition of growth media (Ham's F12 medium supplemented with $10 \%$ FBS), cells were spun down and cell pellet was resuspended in growth media. The cell suspension was passed through eight layers of lens paper and centrifuged at $1,000 \times g$ for $5 \mathrm{~min}$. The cell pellet was dispersed in fresh growth media and cell number was counted with a hemacytometer. The cells were plated at a density of $4 \times 10^{5}$ cells/plate or $1.2 \times 10^{6}$ cells/plate, $35 \mathrm{~mm}$ or $60 \mathrm{~mm}$ in diameter, respectively. The cells were grown at $37^{\circ} \mathrm{C}$ in a $5 \% \mathrm{CO}_{2}$ atmosphere of humidified air. Some of the cultures were treated with $5 \times 10^{9}$ M staurosporine or $1 \mu \mathrm{g} / \mathrm{ml}$ cytochalasin D after cells were 
allowed to attach to the dish for $1 \mathrm{hr}$ at $37^{\circ} \mathrm{C}$ in $5 \% \mathrm{CO}_{2}$.

\section{Immunofluorescence}

Cells were fixed with $4 \%$ paraformaldehyde in PBS for 10 min, washed three times with PBS, permeabilized with 0.1 $\%$ Triton X-100 in PBS for 5 min at room temperature. After washing three times in PBS, cells were blocked with $1 \%$ BSA for $1 \mathrm{~h}$ at room temperature. Incubation with collagen type II antibody $(1: 1,000$, Santa Cruz Biotechnology, Santa Cruz, CA) was performed in blocking solution (1\% BSA in PBS) for 1 hour at room temperature in a humidified chamber. Specimens were washed three times with PBS and incubated for 1 hour at room temperature with Alexa fluor 555-conjugated mouse anti-goat antibody. Typically, we incubated cells with secondary antibody and Alexa fluor 488- conjugated phalloidin simultaneously to examine expression of collagen type II and distribution of F-actin. Nuclei were stained with 4,6-diamidino-2-phenylindole (DAPI). Samples were mounted in mounting medium (Sigma, Saint Louis, MO) and observed using a confocal laser scanning microscopy (LSM700; Carl Zeiss, Oberkochen, Germany).

\section{Real-time quantitative RT-PCR}

Total cellular RNA was extracted with Trizol (Gibco Invitrogen) at various time points. cDNA was synthesized from $1 \mu \mathrm{g}$ RNA in $20 \mu \mathrm{l}$ of master mix for reverse transcription containing $200 \mathrm{U} / \mu$ l Superscript III (Invitrogen), 5 $\mathrm{mM} \mathrm{MgCl}$, PCR Buffer, $1 \mathrm{mM}$ dNTP, $1 \mathrm{U} / \mu \mathrm{l}$ RNase inhibitor, and $2.5 \mathrm{mM}$ oligo dT in DEPC-treated distilled water. For assessment of mRNA, transcripts were quantified by real-time quantitative polymerase chain reaction (RT-PCR) and normalized with respect to gapdh expression. The oligonucleotides used as primers were as follows: Type II collagen; 5'-TGGATATTGGCCCAGTCTG CTTCT-3', antisense, 5'-TGGGATTGGGCTGGATTCCTTT CT-3' sense, gapdh; 5'-GATGGGTGTCAACCATGAGAA A-3', antisense', 5'-ATCAAAGGTGGAAGAATGGCTG-3', sense.

\section{Western blot}

Cells were harvested and cell lysates were prepared with cell lysis buffer (50 mM Tris, $\mathrm{pH} 7.4,150 \mathrm{mM} \mathrm{NaCl}, 1 \mathrm{mM}$ EDTA, $1 \mathrm{mM}$ PMSF, $1 \mathrm{mM} \mathrm{NaF}, 1 \mathrm{mM}$ sodium orthovanadate, $0.25 \%$ sodium deoxycholate, and $1 \%$ NP- 40 , protease inhibitor cocktail [Sigma]). Cell extracts were cleared by centrifugation at $15,000 \times g$ for $10 \mathrm{~min}$ at $4^{\circ} \mathrm{C}$ and the protein concentration of each sample was measured by the BCA protein assay (Pierce, Rockford, IL). Equal amount of lysates were subjected to $10 \%$ SDS-PAGE and electroblotted to nitrocellulose membrane. Blots were blocked with $3 \%$ non-fat dry milk in TBST buffer $(20 \mathrm{mM}$ Tris- $\mathrm{HCl}$, $150 \mathrm{mM} \mathrm{NaCl}, 0.1 \%$ Tween-20) for $1 \mathrm{~h}$ and incubated with primary antibodies against type II collagen, cofilin, phospho-cofilin, MLC, phosphor-MLC overnight at $4^{\circ} \mathrm{C}$. Appropriate secondary antibodies coupled to horseradish peroxidase were then applied at a 1:1,000 dilution for $1 \mathrm{~h}$ at room temperature. Proteins were detected using Supersignal Femto Maximum Sensitivity Substrate (Pierce). Quanti- fication of relative band densities was performed by scanning densitometry using Image J software (National Institute of Health, Bethesda, MD).

\section{Measurement of F-actin/G-actin ratio}

F-actin to G-actin ratio was determined as described previously (Rasmussen et al., 2010). Briefly, cells were lysed with actin stabilization buffer (0.1 M PIPES, pH 6.9, 30\%

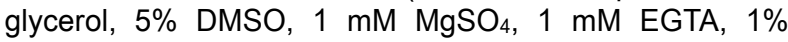
TX-100, $1 \mathrm{mM}$ ATP, protease inhibitor cocktail (Sigma)) on ice for 10 minutes. Cells were harvested and the cell extracts were centrifuged at $4^{\circ} \mathrm{C}$ for 75 minutes at $16,000 \mathrm{~g}$ to separate the F-actin from $\mathrm{G}$-actin pool. The supernatants of the extracts were collected and designated a G-actin pool. The pellets were resuspended in ice-cold actin depolymerization buffer (0.1 M PIPES, pH 6.9, $1 \mathrm{mM} \mathrm{MgSO}_{4}$, $10 \mathrm{mM} \mathrm{CaCl}_{2}$, and $5 \mu \mathrm{M}$ cytochalasin $\mathrm{D}$ ) and designated a $\mathrm{F}$-actin pool. Equal amounts of both the supernatant (G-actin) and the resuspended pellet (F-actin) were subjected to Western blot with the use of an anti- $\beta$-actin antibody.

\section{Acknowledgements}

This Research was supported by Kyungpook National University Research Fund, 2009.

\section{References}

Agnew BJ, Minamide LS, Bamburg JR. Reactivation of phosphorylated actin depolymerizing factor and identification of the regulatory site. J Biol Chem 1995;270:17582-7

Ahrens PB, Solursh M, Reiter RS. Stage-related capacity for limb chondrogenesis in cell culture. Dev Biol 1977;60:69-82

Amano M, Nakayama M, Kaibuchi K. Rho-kinase/ROCK: a key regulator of the cytoskeleton and cell polarity. Cytoskeleton 2010;67:545-54

Bamburg JR, Bernstein BW. Roles of ADF/cofilin in actin polymerization and beyond. F1000 Biol Rep 2010;2:62

Benya PD, Padilla SR. Modulation of the rabbit chondrocyte phenotype by retinoic acid terminates type II collagen synthesis without inducing type I collagen: the modulated phenotype differs from that produced by subculture. Dev Biol 1986;118:296-305

Bershadsky AD, Gelfand VI, Svitkina TM, Tint IS. Destruction of microfilament bundles in mouse embryo fibroblasts treated with inhibitors of energy metabolism. Exp Cell Res 1980;127:421-9

Bonder EM, Mooseker MS. Cytochalasin B slows but does not prevent monomer addition at the barbed end of the actin filament. J Cell Biol 1986;102:282-8

Borge L, Lemare F, Demignot S, Adolphe M. Restoration of the differentiated functions of serially passaged chondrocytes using staurosporine. In Vitro Cell Dev Biol Anim 1997; 33:703-9

Brown PD, Benya PD. Alterations in chondrocyte cytoskeletal architecture during phenotypic modulation by retinoic acid 
and dihydrocytochalasin B-induced reexpression. J Cell Biol 1988;106:171-9

Chicurel ME, Singer RH, Meyer CJ, Ingber DE. Integrin binding and mechanical tension induce movement of mRNA and ribosomes to focal adhesions. Nature 1988;392:730-3

Cooper JA. Effects of cytochalasin and phalloidin on actin. J Cell Biol 1987;105:1473-8

DeLise AM, Fischer L, Tuan RS. Cellular interactions and signaling in cartilage development. Osteoarthritis Cartilage 2000;8:309-34

Etienne-Manneville S, Hall A. Rho GTPases in cell biology. Nature 2002;420:629-35

Hoben GM, Athanasiou KA. Use of staurosporine, an actin-modifying agent, to enhance fibrochondrocyte matrix gene expression and synthesis. Cell Tissue Res 2008; 334:469-76

Huang TY, DerMardirossian C, Bokoch GM. Cofilin phosphatases and regulation of actin dynamics. Curr Opin Cell Biol 2006;18:26-31

Janmey PA. The cytoskeleton and cell signaling: component localization and mechanical coupling. Physiol Rev 1998;78: 763-81

Kim MJ, Kim S, Kim Y, Jin EJ, Sonn JK. Inhibition of RhoA but not ROCK induces chondrogenesis of chick limb mesenchymal cells. Biochem Biophys Res Commun 2012;418: 500-5

Kumar D, Lassar AB. The transcriptional activity of Sox9 in chondrocytes is regulated by RhoA signaling and actin polymerization. Mol Cell Biol 2009;29:4262-73

Lee CR, Grodzinsky AJ, Spector M. Modulation of the contractile and biosynthetic activity of chondrocytes seeded in collagen-glycosaminoglycan matrices. Tissue Eng 2003; 9:27-36

Lim YB, Kang SS, Park TK, Lee YS, Chun JS, Sonn JK. Disruption of actin cytoskeleton induces chondrogenesis of mesenchymal cells by activating protein kinase C-alpha signaling. Biochem Biophys Res Commun 2000;273:609-13

Loty S, Forest N, Boulekbache H, Sautier JM. Cytochalasin $D$ induces changes in cell shape and promotes in vitro chondrogenesis: a morphological study. Biol Cell 1995;83: 149-61

Maekawa M, Ishizaki T, Boku S, Watanabe N, Fujita A, Iwamatsu A, Obinata T, Ohashi K, Mizuno K, Narumiya S. Signaling from Rho to the actin cytoskeleton through protein kinases ROCK and LIM-kinase. Science 1999;285:895-8

Matsumoto H, Sasaki Y. Staurosporine, a protein kinase $\mathrm{C}$ inhibitor interferes with proliferation of arterial smooth muscle cells. Biochem Biophys Res Commun 1989;158: 105-9

Moriyama K, lida K, Yahara I. Phosphorylation of Ser-3 of cofilin regulates its essential function on actin. Genes Cells 1996;1:73-86

Nobes CD, Hall A. Rho, rac, and cdc42 GTPases regulate the assembly of multimolecular focal complexes associated with actin stress fibers, lamellipodia, and filopodia. Cell 1995;81:

\section{3-62}

Paavilainen VO, Bertling E, Falck S, Lappalainen P. Regulation of cytoskeletal dynamics by actin-monomer-binding proteins. Trends Cell Biol 2004;14:386-94

Pollard TD, Cooper JA. Actin, a central player in cell shape and movement. Science 2009;326:1208-12

Rasmussen I, Pedersen LH, Byg L, Suzuki K, Sumimoto H, Vilhardt F. Effects of F/G-actin ratio and actin turn-over rate on NADPH oxidase activity in microglia. BMC Immunology 2010;11:44-8

Rottner K, Hall A, Small JV. Interplay between Rac and Rho in the control of substrate contact dynamics. Curr Biol 1999;9:640-8

Rueckschloss $\mathrm{U}$, Isenberg $\mathrm{G}$. Cytochalasin $\mathrm{D}$ reduces $\mathrm{Ca2}^{+}$ currents via cofilin-activated depolymerization of F-actin in guinea-pig cardiomyocytes. J Physiol 2001;537:363-70

Schliwa M. Action of cytochalasin D on cytoskeletal networks. J Cell Biol 1982;92:79-91

Scott RW, Olson MF. LIM kinases: function, regulation and association with human disease. J Mol Med 2007;85:555-68

Solursh M, Linsenmayer TF, Jensen KL. Chondrogenesis from single limb mesenchyme cells. Dev Biol 1982;94:25964

Tamaoki T, Nomoto H, Takahashi I, Kato Y, Morimoto M, Tomita F. Staurosporine, a potent inhibitor of phospholipid/Ca ${ }^{++}$dependent protein kinase. Biochem Biophys Res Commun 1986;135:397-402

Tortelli F, Cancedda R. Three-dimensional cultures of osteogenic and chondrogenic cells: a tissue engineering approach to mimic bone and cartilage in vitro. Eur Cell Mater 2009;17: 1-14

Toshima J, Toshima JY, Takeuchi K, Mori R, Mizuno K. Cofilin phosphorylation and actin reorganization activities of testicular protein kinase 2 and its predominant expression in testicular Sertoli cells. J Biol Chem 2001;276:31449-58

Totsukawa G, Yamakita Y, Yamashiro S, Hartshorne DJ, Sasaki Y, Matsumura F. Distinct roles of ROCK (Rho-kinase) and MLCK in spatial regulation of MLC phosphorylation for assembly of stress fibers and focal adhesions in 3T3 fibroblasts. J Cell Biol 2000;150:797-806

Vicente-Manzanares M, Ma X, Adelstein RS, Horwitz AR. Non-muscle myosin II takes centre stage in cell adhesion and migration. Nat Rev Mol Cell Biol 2009;10:778-90

von der Mark K, von der Mark H. Immunological and biochemical studies of collagen type transition during in vitro chrondrogenesis of chick limb mesodermal cells. J Cell Biol 1977;73:736-47

Watanabe N, Kato T, Fujita A, Ishizaki T, Narumiya S. Cooperation between mDia1 and ROCK in Rho-induced actin reorganization. Nat Cell Biol 1999;1:136-43

Wezeman FH. Morphological foundations of precartilage development in mesenchyme. Microsc Res Tech 1998;43: 91-101

Woods A, Wang G, Beier F. RhoA/ROCK signaling regulates Sox9 expression and actin organization during chondro- 
genesis. J Biol Chem 2005;280:11626-34

Yanagihara N, Tachikawa E, Izumi F, Yasugawa S, Yamamoto $\mathrm{H}$, Miyamoto $\mathrm{E}$. Staurosporine: an effective inhibitor for $\mathrm{Ca}^{2+}$ /calmodulin-dependent protein kinase II. J

\section{Neurochem 1991;56:294-8}

Zanetti NC, Solursh M. Induction of chondrogenesis in limb mesenchymal cultures by disruption of the actin cytoskeleton. J Cell Biol 1984;99:115-23 